\title{
Effect of Expansion Pressure on the Drug Eluting Coating and the Corrosion Characteristics of Coronary Stents
}

\author{
Krisztina HORICSÁNYI, ${ }^{1}$ Lilla ASZTALOS, ${ }^{2}$ Dóra KÁROLY, ${ }^{3}$ Éva FAZAKAS ${ }^{4}$ \\ 1,2,3 Budapest University of Technology and Economy, Department of Materials Science \& Engineering, \\ Budapest, Hungary \\ ${ }^{1}$ horicsanyi.krisztina@gmail.com \\ 2 lilla@eik.bme.hu \\ ${ }^{3}$ kdora@eik.bme.hu \\ ${ }^{4}$ Bay Zoltán Nonprofit Ltd. for Applied Research, Budapest, Hungary, eva.fazakas@bayzoltan.hu
}

\begin{abstract}
During implantation, stents are delivered in crimped state to the narrowed lesion, where they are expanded to the desired size by the balloon. Due to insufficient size selection or high resistance to plaque, the stent is often widened by the expansion pressure to a level greater than the nominal pressure specified by the manufacturer. Depending on the degree of overpressure, the nominal diameter of the stent may change by several tenths of a millimetre. Numerous studies have dealt with the physiological effects of overexposure and stenogenic stress, but so far no studies have been carried out to investigate the stent coating and corrosion properties of the stent. In our research a widely used drug-eluting, platinum-chromium alloyed steel stent was observed with an inflation pressure of 12 and 18 bar. Scanning electron microscopy revealed lesions of the coating and potentiodynamic tests were performed to determine the corrosion rate.
\end{abstract}

Keywords: coronary stent, corrosion, expansion pressure, platinum-chromium alloyed steel, drug eluting stent.

\section{The overexpansion of coronary stents}

Due to inadequate size selection or high resistance of plaque, in order to maintain the stent diameter to the desired extent, the stents are often expanded to a greater than nominal pressure during intervention. During stent implantation, the vascular wall is damaged and therefore stents are not able to completely reduce the neointimal proliferation induced by vascular injury during implantation [1]. Enlargement above the nominal as first recommended for Palmaz-Schatz stents, to optimize stent cleavage and reduce subacute stent thrombosis [2].

The overtraining is not always done immediately, but can also be used to determine whether the stem is originally prone to further expansion [3].
There are several research studies on the physiological effects of stents overdosing [1, 2, 4], but few of the effects on its functional properties are available [3].

\section{Materials and methods}

The aim of our research was to investigate the coat damping of two similar size and type of coronary artery stent from the same manufacturer but expanded with different pressure values and to observe the effect of the expansion pressure on the corrosion rate.

Before and after the expansion on the balloon and without the balloon stereomicroscopic images were taken to determine the degree of radial recoil. The condition of the stents removed from the balloon was checked by scanning electron microscopy. Potentiometric measurements were 
performed in a three electrode cell corresponding to MSZ EN ISO 10993-15: 2009 for the corrosion rate at $37 \pm 1{ }^{\circ} \mathrm{C}$ in a $0.9 \mathrm{~m} / \mathrm{m} \%$ isotonic saline solution. The auxiliary electrode was platinum mesh and the reference electrode was $\mathrm{Hg} / \mathrm{Hg}_{2} \mathrm{Cl}_{2}, \mathrm{KCl}$ calomel electrode. The measuring range was determined at $\pm 250 \mathrm{mV}$.

\section{Results}

\subsection{Stereomicroscopic measures}

The two platinum-chromium alloyed everolimus coated stents were a size of

$2.25 \times 32 \mathrm{~mm}$ and expanded by 12 bar (nominal) and 18 bar (max). Stereomicroscopic images were taken in the expanded state on the expanded balloon and also after removing it, so that we could determine the degree of radial recoil. Recoil is the value by which the diameter of the implant changes from the initial diameter (when it is on the fully inflated balloon) to the final, resting diameter, expressed as a percentage. Table 1 shows the measured and calculated values of the two stents. The $32 \mathrm{~mm}$ stent consists of 38 rings, the values shown in the table are the average of the diameter of each ring.

Table 1. The average diameter measured on the specimens and the degree of radial recoil

\begin{tabular}{|l|c|c|}
\hline & 12 bar & 18 bar \\
\hline On the balloon & $2.380 \mathrm{~mm}$ & $2.610 \mathrm{~mm}$ \\
\hline Without balloon & $2.304 \mathrm{~mm}$ & $2.585 \mathrm{~mm}$ \\
\hline Values from datasheet & $2.25 \mathrm{~mm}$ & $2.5 \mathrm{~mm}$ \\
\hline Recoil & $3.16 \%$ & $0.97 \%$ \\
\hline
\end{tabular}

It can be seen from the data that the stent is larger in diameter than the stent data sheet both in nominal and over expanded state. It can be stated that by increasing the expansion pressure the radial recoil rate has decreased and the stent diameter can be increased by more than $10 \%$ relative to the nominal data.

\subsection{Scanning electron microscope exami- nations}

The purpose of the scanning electron microscopic tests was to detect damage on the stent coatings. Since the stent coating is made up of the active substance (everolimus) and a polymer matrix, therefore, backscattered electron detection was used since heavy elements (e.g. metals) back- scatter electrons more strongly than light elements, and thus appear brighter in the image. At the lighter parts of the stent the metallic base can be seen which indicates severe coating damage.

With overpressure, we have found major injuries on almost every ring. At a nominal pressure only on every 4-5 ring, coating damage was found where the metallic surface was already directly visible.

In addition to the number of documented injuries, their size is significantly larger for the over expanded stent: up to 100-120 $\mu$ m (Figures 1, 2), while the average size of the nominal expanded sample is 20-30 $\mu \mathrm{m}$ (Figures 3, 4).

In the area indicated in Figure 1, the coating is not just split but also creased. This kind of crease of the coating was observed in several places on the nominal pressure expanded stent also.

In the case of the over expanded sample, in several places we observed not only individual injuries, but also groups of smaller injuries (Figure 5).

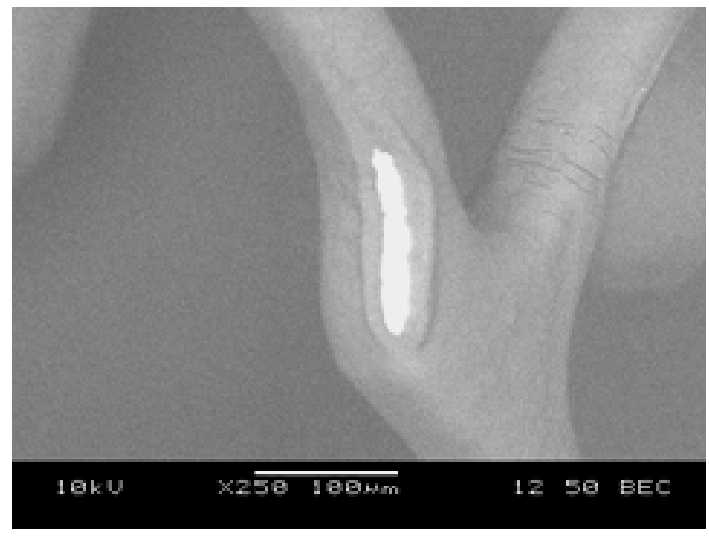

Figure 1. Coating damage on the 18 bar expanded stent

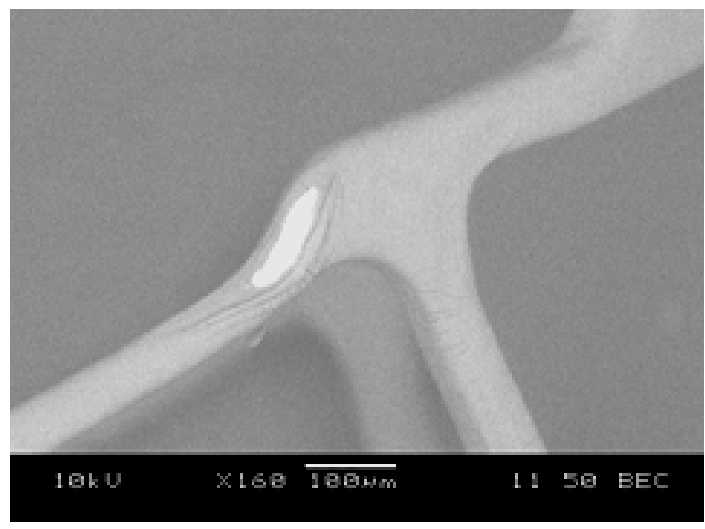

Figure 2. Coating damage on the 18 bar expanded stent. 


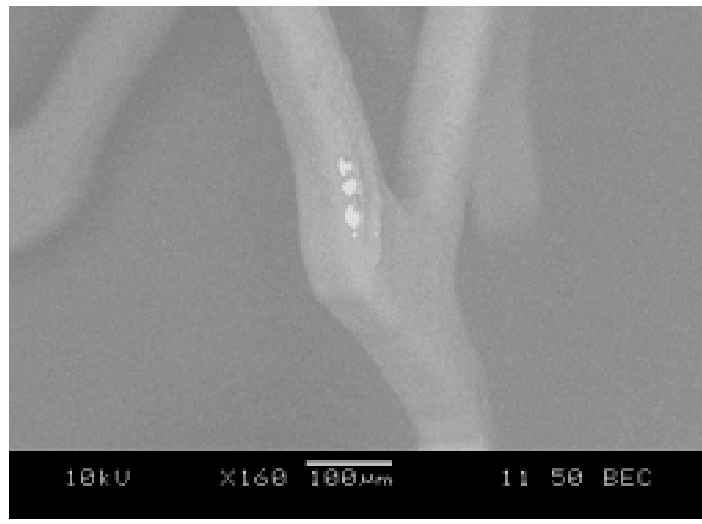

Figure 3. Coating damage on the 12 bar expanded stent.

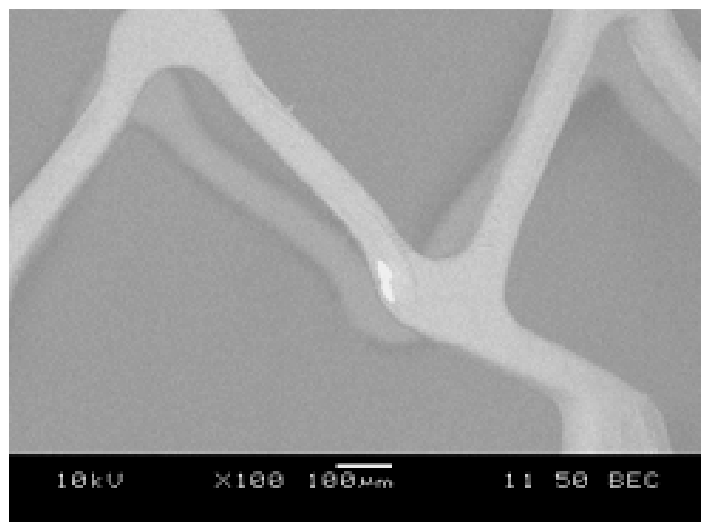

Figure 4. Coating damage at the 12 bar expanded stent.

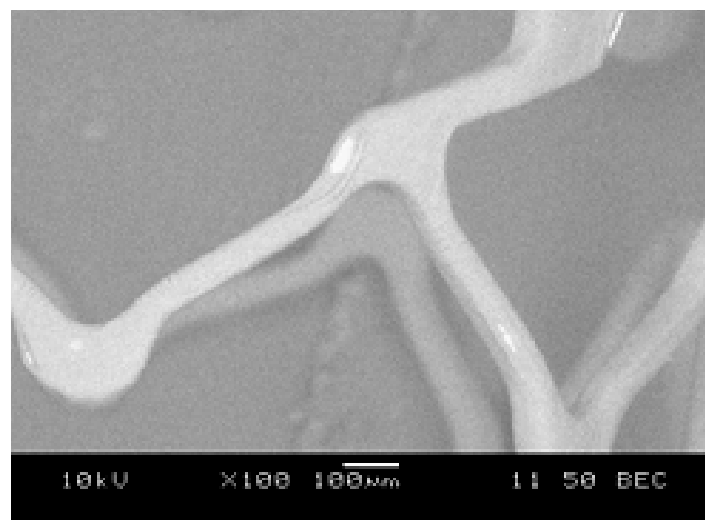

Figure 5. Group of coating injuries on the 18 bar expanded stent

Table 2. The corrosion rate calculated for the samples at the start of the measurement and after 2 hours in $\mu \mathrm{m} / \mathrm{year}$

\begin{tabular}{|c|c|c|c|}
\hline \multicolumn{2}{|c|}{12 bar } & \multicolumn{2}{c|}{18 bar } \\
\hline 0 hour & 2 hours & 0 hour & 2 hours \\
\hline 0.0154 & 0.0096 & 0.0118 & 0.0090 \\
\hline
\end{tabular}

\subsection{Corrosion tests}

Potentiometric measurements were made to determine the corrosion rate according to MSZ EN ISO 10993-15: 2009. Before and after the corrosion test, the mass of stents was also measured.

As shown in Table 2, the corrosion rate of the over expanded stent - not just at the beginning of the measurement but also 2 hours later was the more favorable, but the corrosion rate of the nominal expansion stent also indicates very good corrosion properties. The mass of the two stents remained unchanged during the measurements.

\section{Conclusions}

The effect of over expanding of stents on the functional properties is a small area under consideration despite the fact that in clinical practice, expansion with greater than nominal pressure is often encountered. In our research, the degree of radial recoil, coating injuries and corrosion rates were investigated in two coronary arteries of the same size and type.

It can be stated that by increasing the expansion pressure, a significant increase in diameter (more than $10 \%$ ) can be achieved, but we could also get higher diameter values both at nominal and at over expansion - as shown in the manufacturer's data sheet. The recoil decreased to one third by overexpanding, with a recoil rate of $0.97 \%$ at 18 bar.

The over expanded stent was heavily damaged, with almost every ring having smaller or bigger seizures, compared to the nominal pressured case where only every 4-5. ring displayed visible damage. The "crease" of the coating can be observed on both stents in several places.

The corrosion rate at the end of both the initial and the 2-hour measurements is more favorable for the over expanded stent.

\section{Acknowledgments}

Acknoledgment for the financial support given by the project the ÚNKP-17- 3-II new national excellence program of the ministry of human capacities

\section{References}

[1] Berrocal D. H., González G. E., Fernández A., Perez S., Wilensky L., Morales C., Grinfeld L., Gelpi R.J.: Effects of overexpansion on stents' recoil, symmetry/asymmetry, and neointimal hyperplasia in aortas of hypercholesterolemic rabbits. Cardiovascular Pathology, 17/5. (2008) 289-296. https://doi.org/10.1016/j.carpath.2007.10.005 
[2] Hoffmann R., Haager P., Mintz G.S., Kerckhoff G., Schwarz R., Franke A., Vom Dahl J., Hanrath P.: The impact of high pressure vs low pressure stent implantation on intimal hyperplasia and follow-up lumen dimensions. European Heart Journal, 22/21. (2001) 2015-2024.

https://doi.org/10.1053/euhj.2001.2609

[3] Jaryl N., Foin N., Hui Ying Ang, Jiang Ming Fam, Sayan Sen, Sukhjinder Nijjer, Petraco R., Di Mario C., Davies J., Wong P.: Over-expansion capacity and stent design model: An update with contem- porary DES platforms. International Journal of Cardiology, 221. (2016) 171-179.

https://doi.org/10.1016/j.ijcard.2016.06.097

[4] Kuriyama N., Yoshio Kobayashi, Nakabumi Kuroda, Kartik Desai, Yutaka Yamamoto, Nobuyuki Komiyama, Issei Komuro, Fitzgerald P. J.: Effect of coronary stent overexpansion on lumen size and intimal hyperplasia at follow-up. The American Journal of Cardiology, 89/11. (2002) 1297-1299. https://doi.org/10.1016/S0002-9149(02)02330-5 